\title{
Accurate Calculation of Partial Inductances for the Orthogonal PEEC Formulation
}

\section{Journal Article}

\section{Author(s):}

Kovacevic-Badstuebner, Ivana; Romano, Daniele; Lombardi, Luigi; Grossner, Ulrike (D); Ekman, Jonas; Antonini, Giulio

Publication date:

2021-02

Permanent link:

https://doi.org/10.3929/ethz-b-000456672

Rights / license:

In Copyright - Non-Commercial Use Permitted

Originally published in:

IEEE Transactions on Electromagnetic Compatibility 63(1), https://doi.org/10.1109/TEMC.2020.2986933 


\title{
Accurate Calculation of Partial Inductances for the Orthogonal PEEC Formulation
}

\author{
Ivana Kovačević-Badstübner, Member, IEEE, Daniele Romano, Luigi Lombardi, Ulrike Grossner, Member, IEEE, \\ Jonas Ekman Member, IEEE, and Giulio Antonini, Senior Member, IEEE,
}

\begin{abstract}
The Partial Element Equivalent Circuit (PEEC) method is promising numerical technique for three-dimension electromagnetic modeling across various application fields. In the framework of the PEEC method, the partial elements modeling the magnetic and electric field coupling between elementary volumes and surfaces are computed by double-folded volume and surface integrals. Assuming the quasi-static hypothesis and an orthogonal mesh, the integrals have been computed by the analytical formulas derived in literature, which significantly reduces the computational time in comparison to the numerical integration. However, the existing analytical formulas are affected by significant numerical errors for certain PEEC structural mesh necessary to model the skin and proximity effects with a higher accuracy. To utilize the full potential of the PEEC method, the calculation of partial elements has to be carefully addressed, which has not been investigated in a comprehensive way so far. Accordingly, this paper presents a systematic accuracy analysis of the existing closed-form analytical formulas and methods for calculating the self and mutual inductances between two rectangular conductors. Additionally, a new strategy to select a proper analytical formula depending on the dimensions and positions of two conductors is proposed, which allows the mutual inductance extraction with a relative error of less than $0.1 \%$. The new method is systematically validated on examples of 3D dense PEEC systems using the quadruple precision arithmetic as reference.
\end{abstract}

Index Terms-Integral equations, electric field, adaptive integration, numerical integration, partial element equivalent circuit (PEEC) method.

\section{INTRODUCTION}

With the trends towards higher integration and enhanced system performance, the design of interconnections in electronic systems becomes a challenging task that should be addressed at a very early design stage. Electromagnetic modeling (EM) therefore increases in importance as it allows engineers to extract parasitics and hence, optimize the interconnections

Manuscript received October, 2019. Corresponding author: G. Antonini (email: giulio.antonini@univaq.it).

Daniele Romano and Giulio Antonini are with the UAq EMC Laboratory, Department of Industrial and Information Engineering and Economics, University of L'Aquila, I-67100 L'Aquila, Italy, (e-mail: daniele.romano.vis@gmail.com; giulio.antonini@univaq.it).

Ivana Kovačević-Badstübner, Ulrike Grossner are with the Advanced Powe Semiconductor Laboratory, ETHZ, Zurich, Switzerland, (e-mail: kovacevic@aps.ee.ethz.ch, ulrike.grossner@ethz.ch).

Luigi Lombardi is with Micron Semiconductor Italia S.r.l, 67051 Avezzano (AQ), Italy (e-mail: luigilombardi89@gmail.com).

Jonas Ekman is with Luleå University of Technology, Sweden, (e-mail: jonas.ekman@ltu.se). in advance to rather expensive hardware prototyping, which is of interest in different application fields as e.g. power electronics, integrated circuits, signal integrity (SI), and radio frequency (RF) applications. For the systems operating across a wide frequency range including both dc, steady-state and dynamic operation, accurate and computationally efficient EM simulations is not straightforward. For example, for future power electronic applications, the switching frequency tends to increase from $\mathrm{kHz}$ to $\mathrm{MHz}$ range [1]; on the other hand the frequencies contained in the switching transients of advanced power semiconductor devices belong to the range of hundreds $\mathrm{MHz}$. Additionally, in comparison to RF and SI applications, where the modeling domain can be often approximated in two dimensions, the modeling domains in power electronics applications have to be typically treated as three-dimensional (3D) geometries, as e.g. power semiconductor packages. In the 3D domain, the EM modeling tools have to be able to accurately capture the high frequency effects, i.e. skin and proximity effects, in a wide frequency range. Commercially available EM modeling tools based on different numerical techniques offer the numerical solvers specialized either for low frequency (LF) or high frequency (HF) domain [2]-[4]. For modeling from DC to HF frequencies, two numerical solvers have to be used, which requires the results to be properly merged [5]; hence, using a single numerical technique in the whole frequency range is a more attractive approach. In this respect, the Partial Element Equivalent Circuit (PEEC) method [6], [7] is a promising numerical technique for 3D EM modeling across various applications as e.g. power electronics. The advantage of the PEEC-based modeling of 3D geometries is a circuit representation of electromagnetic properties without modeling the air/vacuum region surrounding conductive, dielectric and magnetic bodies. Namely, the PEEC method is a full-wave method and it is applicable for modeling both quasi-static EM fields and EM wave propagation. The electric and magnetic field effects and their coupling are modeled by the partial elements including partial inductances, partial resistances and coefficients of potentials.

3D modeling structures can be frequently treated as electromagnetically small, so that the exponential term appearing in the free-space Green's function can be approximated as a unit neglecting the EM wave propagation delays. For this case, the quasi-static approximation is valid and the PEEC system matrix is formed by three matrices: the diagonal $\mathbf{R}$ matrix modeling ohmic losses, and the dense symmetric matrices, $\mathbf{L}_{\mathrm{p}}$ and $\mathbf{P}$, modeling magnetic field and electric field effects, 
respectively. The two-way coupling between magnetic and electric fields, is inherently included with the PEEC method in comparison to the quasi-static EM modeling as implemented in e.g. Fast-Henry and ANSYS Q3D extractor. The main challenge of the PEEC modeling is a very dense system of equations, which implies the requirements for higher memory storage and computational power. Additionally, the accuracy of $\mathbf{R}, \mathbf{L}_{\mathrm{p}}$ and $\mathbf{P}$ matrix elements has a significant impact on the overall accuracy of PEEC-based 3D modeling when a dense PEEC system matrix has to be solved [8], [9]. With all these challenges, the PEEC method is still behind the matured techniques such as the Finite Element Method with respect to its implementation in commercially available EM modeling environments. To utilize the full potential of the PEEC method, the accuracy of partial element calculation has to be addressed first.

The starting point of the PEEC modeling is discretization of the 3D structures and calculation of $\mathbf{R}, \mathbf{L}_{\mathrm{p}}$ and $\mathbf{P}$ matrix elements. For orthogonal structural meshing, i.e. simplifying complex geometries with the stair-case approximation where the discretization cells are defined by rectangular surfaces and rectangular parallelepipeds with the edges either parallel or orthogonal to each other, the closed-form analytical formulas for $L_{\mathrm{p}}$ - and $P$-integrals can be used. This leads to significantly faster calculation time of $\mathbf{L}_{p}$ and $\mathbf{P}$ matrix elements [9]. On the other hand, to take more complex geometrical structures into account, partial elements are typically extracted by numerical integration [8], [10]-[12], which in turn negatively affects the computational time. Therefore, the geometry simplification is preferred as long as the stair-case approximation of 3D structures does not introduce significant errors and faster calculation time is beneficial as e.g. large-scale problems and the optimization routines.

The main advantage of these analytical formulas is that they provide, ideally, a rigorous solution for the integrals defining $\mathbf{L}_{\mathrm{p}}$ and $\mathbf{P}$ PEEC elements. However, in practice, the implementation of such closed form is based on finite numerical structures, typically double precision floating point (IEEE ${ }^{\circledR}$ Standard 754), which introduce a truncation error. It is known that for cells with extreme form factors (the ratios between the sizes of the cells) or for extreme ratio distance/size, the truncation error might be so large that the computation of the partial elements is completely dominated by the numerical error [7], [9], [13], [14]. The numerical errors come from summing the terms of different orders appearing in the closedform formulas using the standard double precision arithmetic, i.e. 16 digits. With the current 64-bit architecture, calculating the partial elements using an extended representations for the real number (e.g. quadruple precision defined by 32 digits) is too slow for the computation of the large matrices needed for an accurate solution of practical EM problems. This makes the PEEC method less attractive for various practical applications. Solving integrals by numerical integration on the other hand requires a higher order of integration for the cells at the small mutual distance and hence, implied higher computational cost. Particularly, with respect to the number of unknowns, the quadruple precision can be adopted for the calculation of diagonal matrix elements (self-terms), however, the double precision arithmetic is preferred for the calculation of offdiagonal terms describing the mutual couplings. Therefore, a lot of efforts have been made to develop improved analytical formulas for the extraction of mutual inductance between two orthogonal conductors tending to achieve high accuracy even for the special cases as e.g. the large aspect ratio volumes and far-away volumes. It should be noted that the mutual $\mathbf{P}$ elements can be derived in the second step from the formulas used for $\mathbf{L}_{\mathrm{p}}$ elements calculation.

The analytical formulas proposed in literature [13], [14] were only verified for a limited number of examples of two rectangular conductors and a more thorough verification of these formulas is missing to derive general conclusions. Therefore, this paper investigates the accuracy of the available improved formulas [13], [14] in a comprehensive way using carefully selected examples of rectangular conductors pairs, which could occur in practice when a fine meshing is needed to accurately model the HF behavior of 3D modeling structures. Additionally, a new strategy for selecting the right analytical formula depending on the position and dimensions of two conductors is proposed allowing the extraction of mutual inductance with the relative error of less than $0.1 \%$. The paper is organized as follows. In Section II, the analytical formulas regarded as the most accurate solutions in literature for the calculation of mutual inductance between two rectangular conductors are presented and their accuracy is validated, pointing out to the main motivation behind the research conducted in this paper. In Section III, a new strategy for solving the existing numerical errors is described and further verified in Section IV in terms of accuracy and computational time on examples of highly dense PEEC modeling systems.

\section{Self And Mutual Inductance Computation}

The well-known analytical formulas for the self- and mutual-partial inductances of conductors with rectangular cross section were first provided in [15]-[18]. In [19], the range of applicability and validity of these formulas was investigated, showing the potential numerical problems when using the formulas for certain geometries. Using the formulas from [16], Piatek et al. analyzed the volume-volume and surface-surface cases of two orthogonal cells in [20], and presented the formulas for the self-inductance of a conductor with rectangular cross section in [21]. Zhong et al. proposed in [13] an improved formula for partial mutual inductance between two rectangular conductors by reducing the calculation of the mutual inductance to a weighted sum of selfinductances of 64 virtual conductors defined by the corner points of two modeled conductors, where the self-inductance formula from [18] (also named as FastHenry formula in [19]) was used. Further, Song et al. demonstrated in [14] that the formula from [13] suffers from the numerical stability errors in the certain cases such as large separation distance between 
the conductors. The authors in [14] derived then a strategy to select an appropriate analytical formula for the mutual inductance between two rectangular conductors (with either parallel or orthogonal edges) of any size and at arbitrary distance by distinguishing the following special cases: volumevolume, volume-surface, surface-surface, surface-line, volumeline, volume-point, surface-point, and line-point. By applying the strategy proposed in [14] on several modeling examples, higher errors can be observed for certain geometrical cases as it is demonstrated and explained in this section. The main aim of the following analysis is to show how it is possible to boost the accuracy of PEEC partial elements without using quadruple precision and numerical integration.

\section{A. Mutual Inductance Calculation}

In the quasi-static PEEC (QS-PEEC) method the magnetic field coupling between two elementary volumes $m$ and $n$ carrying uniform currents is described by the partial inductance which requires the computation of the following double folded volume integral:

$$
L_{p_{n m}}^{(q s, v-v)}=\frac{\mu_{0}}{4 \pi S_{n} S_{m}} \underbrace{\hookrightarrow \mathcal{I}_{v-v}}_{\int_{V_{n}} \int_{V_{m}} \frac{1}{r} d V_{m} d V_{n}}
$$

where $r=\left\|r_{m}-r_{n}\right\|$ is the distance between two points inside the two cells, $S_{m}$ and $S_{n}$ are the cross sections normal to the current directions of two volumes $m$ and $n$, respectively. For far-field interactions, i.e. the center-to-center distance $R_{c c}$ between two cells is large enough, (1) can be written as follows

$$
L_{p_{n m}}^{(q s)}=\frac{\mu_{0}}{4 \pi} \frac{\ell_{n} \ell_{m}}{R_{c c}}
$$

by using the approximation (3)

$$
\tilde{G}_{\text {mean }}^{(q s)}=\frac{1}{V_{n} V_{m}} \int_{V_{n}} \int_{V_{m}} \frac{1}{r} d V_{m} d V_{n} \approx \frac{1}{R_{c c}} .
$$

In (2) $\ell_{m}$ and $\ell_{n}$ denote the sizes of the two cells along their current directions. The derivation of the analytical formulas representing the rigorous solution to (1) is given in [16], [20] for different pairs of geometries as volume-volume, parallel volume-line, surface-surface, parallel surface-line, and parallel line-line. In the following, the main focus is placed on the accuracy of mutual inductance calculation using the improved formula from [13] and the strategy proposed in [14]. The reference results for mutual inductances are generated using the quadruple precision to evaluate the true error of the mutual inductance formulas, which has not been performed in any other analysis presented so far in literature to the best of the authors knowledge.

\section{B. Mutual Inductance Calculation in Special Cases}

Starting from the six variables folded integral (1), a set of $L_{p_{n m}}^{(q s)}$ formulas (4a)-(4i) is developed by suppressing one or more dimensions at the time, which leads to nine special cases: surface-volume $\mathcal{I}_{s-v}$ (4a), surface-surface $\mathcal{I}_{s-s}$ (4b), line-volume $\mathcal{I}_{\ell-v}(4 \mathrm{c})$, point-volume $\mathcal{I}_{p-v}(4 \mathrm{~d})$, line-surface $\mathcal{I}_{\ell-s}(4 \mathrm{e})$, point-surface $\mathcal{I}_{p-s}(4 \mathrm{f})$, line-line $\mathcal{I}_{\ell-\ell}(4 \mathrm{~g})$, pointline $\mathcal{I}_{p-\ell}(4 \mathrm{~h})$, and point-point $\mathcal{I}_{p-p}(4 \mathrm{i})$, where $v, s, \ell$, and $p$ stand for volume, surface, line and point, respectively.

$$
\begin{aligned}
& L_{p_{n m}}^{(q s, s-v)}=\frac{\mu_{0}}{4 \pi S_{n} S_{m}} \ell_{n} \underbrace{\hookrightarrow \mathcal{I}_{s-v}}_{\int_{S_{n}} \int_{V_{m}} \frac{1}{r} d V_{m} d S_{n}} \\
& L_{p_{n m}}^{(q s, s-s)}=\frac{\mu_{0}}{4 \pi S_{n} S_{m}} \ell_{n} \ell_{m} \underbrace{\hookrightarrow \mathcal{I}_{s-s}}_{\int_{S_{n}} \int_{S_{m}} \frac{1}{r} d S_{m} d S_{n}} \\
& L_{p_{n m}}^{(q s, \ell-v)}=\frac{\mu_{0}}{4 \pi S_{n} S_{m}} \ell_{n i} \ell_{n j} \underbrace{\hookrightarrow \mathcal{I}_{\ell-v}}_{\int_{\mathcal{L}_{n}} \int_{V_{m}} \frac{1}{r} d V_{m} d \mathcal{L}_{n}} \\
& L_{p_{n m}}^{(q s, p-v)}=\frac{\mu_{0}}{4 \pi S_{n} S_{m}} \ell_{n i} \ell_{n j} \ell_{n k} \underbrace{\int_{V_{m}} \frac{1}{r} d V_{m}}_{\hookrightarrow \mathcal{I}_{p-v}} \\
& L_{p_{n m}}^{(q s, \ell-s)}=\frac{\mu_{0}}{4 \pi S_{n} S_{m}} \ell_{n i} \ell_{n j} \ell_{m} \underbrace{\longrightarrow \mathcal{I}_{\ell-s}}_{\int_{\mathcal{L}_{n}} \int_{S_{m}} \frac{1}{r} d S_{m} d \mathcal{L}_{n}} \\
& L_{p_{n m}}^{(q s, p-s)}=\frac{\mu_{0}}{4 \pi S_{n} S_{m}} \ell_{n i} \ell_{n j} \ell_{n k} l_{m} \underbrace{\int_{\mathcal{I}_{p}}}_{S_{S_{m}} \frac{1}{r} d S_{m}} \\
& \hookrightarrow \mathcal{I}_{p-s}
\end{aligned}
$$

$$
\begin{aligned}
L_{p_{n m}}^{(q s, p-\ell)} & =\frac{\mu_{0}}{4 \pi S_{n} S_{m}} \ell_{n i} \ell_{n j} \ell_{n k} \ell_{m i} \ell_{m j} \underbrace{\int_{\mathcal{L}_{m}} \frac{1}{r} d \mathcal{L}_{m}}_{\rightarrow \mathcal{I}_{p-\ell}} \\
L_{p_{n m}}^{(q s, p-p)} & =\frac{\mu_{0}}{4 \pi S_{n} S_{m}} \ell_{n i} \ell_{n j} \ell_{n k} \ell_{m i} \ell_{m j} \ell_{m k} \frac{1}{r} .
\end{aligned}
$$

It should be observed that (2) is equivalent to (4i) gained by suppressing all six dimensions, i.e. the case of point-point interaction. In comparison to [16], the special cases of (4e) and $(4 \mathrm{~g})$ for modeling mutually orthogonal line-surface and two orthogonal lines, respectively, besides the parallel cases, are also derived. Particularly, for non-uniform PEEC meshing of 3D structures and without any prior assumptions on current distribution, the mutual inductance of two mesh cells (with parallel current directions) can be approximated using the integrals for the orthogonal surface-line case (cf. Fig. 1) or the orthogonal line-line case in order to improve the accuracy of $L_{p_{n m}}^{(q s)}$ calculation.

The main idea is to approximate two arbitrary orthogonal volume cells by one of these nine cases when the volumevolume analytical formula in the standard precision (16 digits) 


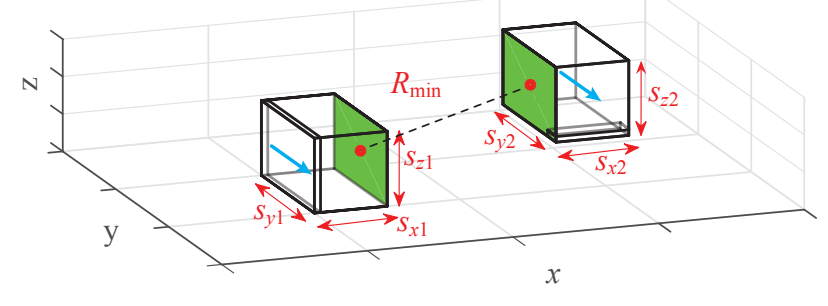

Fig. 1. A pair of volume cells in space with the dimensions $\left(s x_{i}, s y_{i}, s z_{i}\right)$, $i=1,2$, and the distance between cells of $R_{\min }$. If $s x_{1} \ll s y_{1}, s z_{1}$ the first cell can be approximated by a surface in the $y-z$ plane and if $s y_{2}, s z_{2} \ll$ $s x_{2}$ the second cell can be approximated by a line along the $x$ axis. Hence, the surface and the line are orthogonal but the current directions (marked by the arrows) are parallel.

shows a relative error of more than $1 \%$. Song et al. [14] proposed a strategy to select an appropriate analytical formula based on the dimensions and mutual distance of two cells, using a set of derived $L_{p_{n m}}^{(q s)}$ analytical formulas. Similar to [16], the $L_{p_{n m}}^{(q s)}$ analytical formulas derived in [14] depend on the current direction, so that the cases of mutually orthogonal line and surface, and of two orthogonal lines, are not included (considering that the mutual inductance is zero for two orthogonal current directions). Therefore, instead of deriving the analytical form of $L_{p_{n m}}^{(q s)}$ formulas, we derived the rigorous solution of the integrals $\mathcal{I}_{i-j}, i, j=p, \ell, s, v$, as a more general approach. Additionally, two analytical formulas for the orthogonal surface-line case and the orthogonal line-line case omitted in [14] are summarized in Appendix.

The accuracy of the strategy proposed in [14] and the volume-volume analytical formula from [13] is further demonstrated using an example of two volume cells arranged as following:

- the dimensions of the first cell are $s_{x 1}=10 \mu \mathrm{m}, s_{y 1}=$ $4 \mathrm{~mm}, s_{z 1}=0.8 \mu \mathrm{m}$;

- the dimensions of the second cell are $s_{x 2}=1 \mu \mathrm{m}, s_{y 2}=$ $10 \mu \mathrm{m}, s_{z 2}=s x_{2} / k$, where $k$ ranges from $10^{-6}$ to 1 , i.e. $s z_{2}$ varies in the range from $1 \mu \mathrm{m}$ to $1 \mathrm{~m}$;

- the distance between the cells in $x, y$ and $z$ directions are $\Delta x=s_{x 1} \cdot h$, where $h$ ranges from $10^{-2}$ to $10^{5}, \Delta y=0$, $\Delta z=0$ respectively, i.e. the minimum distance between cells $R_{\min }$ varies between $0.1 \mu \mathrm{m}$ and $1 \mathrm{~m}$.

The computation of mutual inductance is performed in the standard double precision arithmetic, while the reference values are computed using the the symbolic math toolbox (with at least 32 digits). The relative errors are estimated in Fig. 2 for the standard analytical formula from [16], $\mathrm{err}_{\text {standard }}$ and the improved analytical formula from [13], $\operatorname{err}_{\text {Zhong. In Fig. }}$ 3 the relative errors for the strategy from [14] based on the condition (5) with two user specified $\epsilon, 10^{-29}$ and $10^{-21}$, and the set of analytical formulas for previously explained nine cases, $\operatorname{err} r_{\text {Song }}$ are shown.

$$
\text { condition }=V_{1} V_{2} \frac{s_{n i} \cdot d_{n 12, \max }}{R_{\min }^{3}}<\epsilon
$$

where $s_{n i}$ is the size of a single cell to be suppressed (either $\left.s_{x i}, s_{y i}, s_{z i}, i=1 . .2\right), d_{n 12, \max }$ is the minimum distance between two cells in the direction of the size being suppressed $(x, y, z), V_{1}$ and $V_{2}$ are the volume of the first and the second cell, and $R_{\min }$ is the distance between the nearest vertices of the two cells, and $\epsilon$ is a very small value.
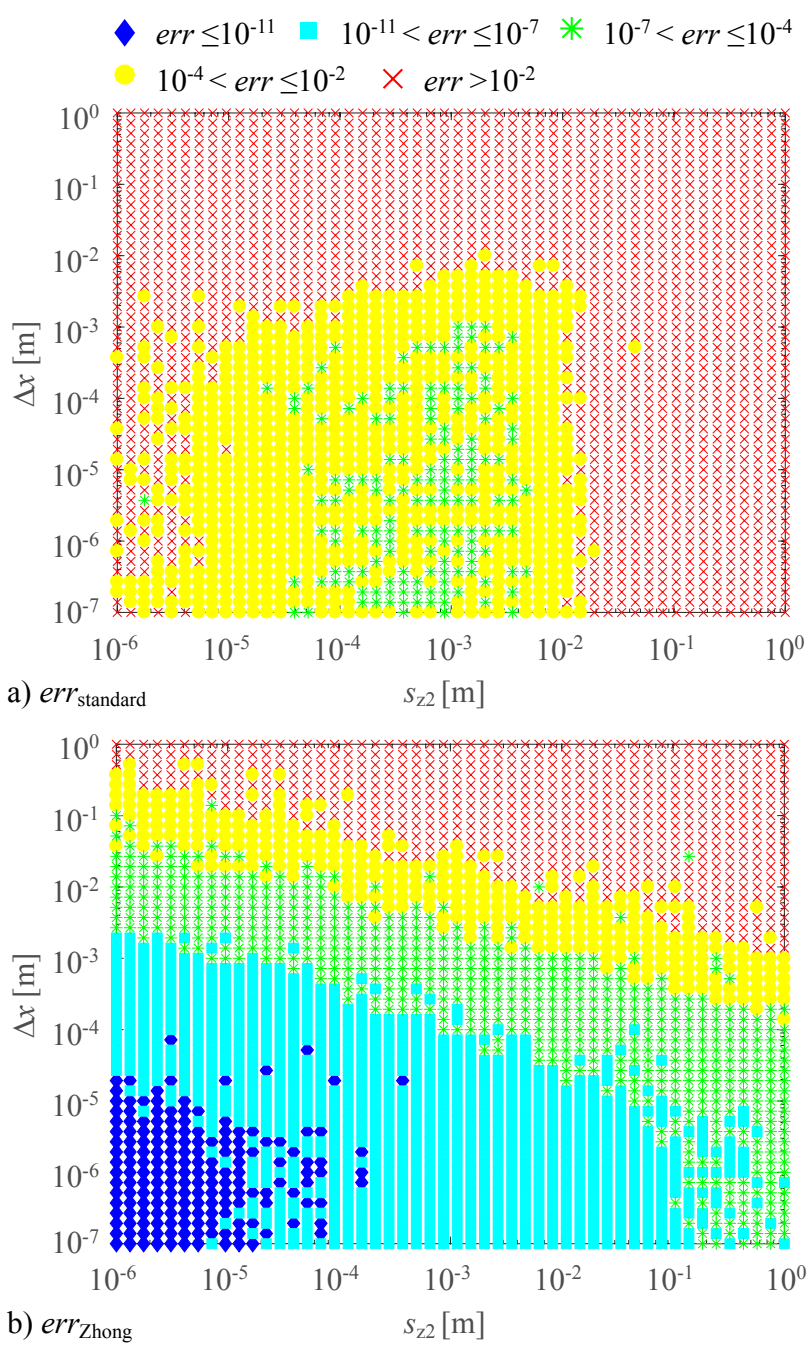

Fig. 2. Relative errors of computing the mutual inductance of two specified cells using a) the standard analytical formula [16], $e r r_{\text {standard, }}$ b) the improved analytical formula from [13], err $r_{\text {Zhong }}$; the distance between two cells is defined by varying $\Delta x$ between $0.1 \mu \mathrm{m}$ and $1 \mathrm{~m}$, and the $z$-dimension of the second cell $s_{z 2}$ varies from $1 \mu \mathrm{m}$ to $1 \mathrm{~m}$.

As it can be seen, the existing methods for calculating the mutual inductance of two selected cells show still the relative error above err $>10^{-2}$, i.e. err $>1 \%$. Specifically, in this experiment, the improved analytical formula from [13] fails for large distances between two volume cells as it was also observed in [14], while the strategy from [14] adds some improvements for higher distances, however still suffers from inaccuracies higher than $1 \%$. Two $\epsilon$ values, $10^{-29}$ 


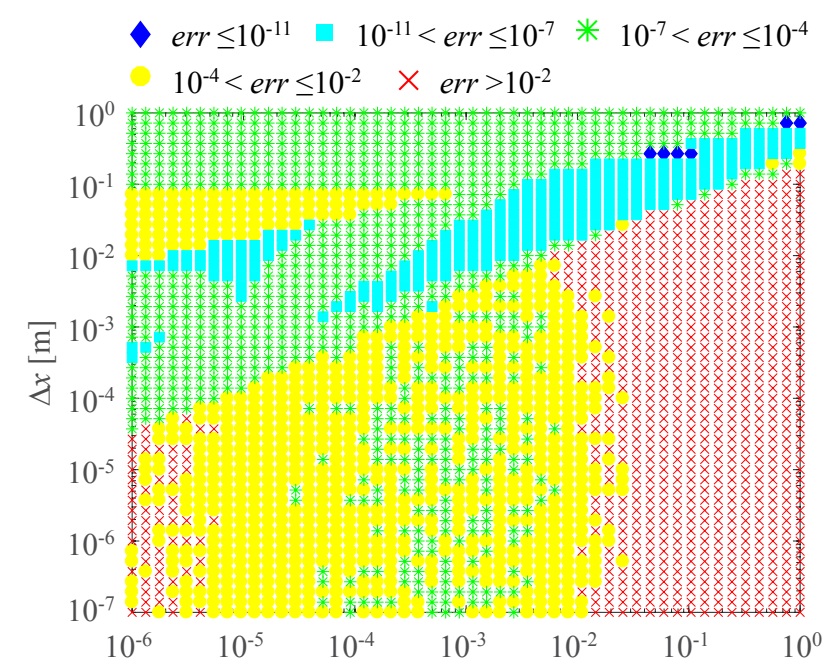

a) $\operatorname{err}_{\text {Song }}\left(\varepsilon=10^{-29}\right) \quad S_{\mathrm{zz}}[\mathrm{m}]$

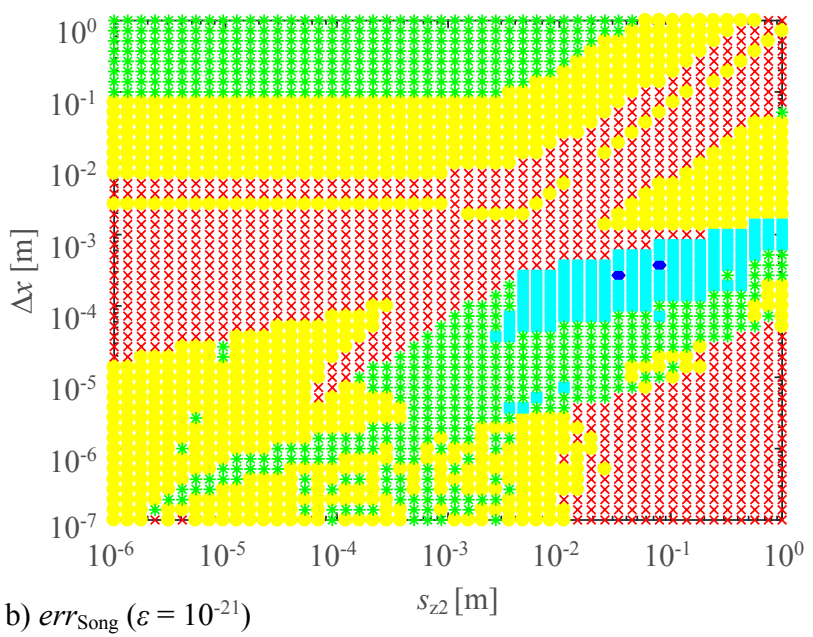

Fig. 3. Relative errors of computing the mutual inductance of two specified cells using the derived analytical formulas based on the strategy proposed in [14], $\operatorname{err}_{\text {Song }}$ with a) $\epsilon=10^{-29}$ and $\epsilon=10^{-21}$; the distance between two cells is defined by varying $\Delta x$ between $0.1 \mu \mathrm{m}$ and $1 \mathrm{~m}$, and the $z$-dimension of the second cell $s_{z 2}$ varies from $1 \mu \mathrm{m}$ to $1 \mathrm{~m}$.

and $10^{-21}$, are selected to show the main challenge of the strategy proposed in [14], i.e. selecting a proper $\epsilon$ value, where $\epsilon=10^{-21}$ was used in [14]. For smaller $\epsilon$, the accuracy gets similar to $e r r_{\text {standard }}$, while for higher $\epsilon$ the accuracy at certain points gets worse. Accordingly, the strategy from [14] requires further improvements, and the main reason for the observed inaccuracies comes from the condition (5), which is modified as described in the next section and Appendix.

\section{Mutual Inductance Calculation: Proposed Strategy}

The non-uniform PEEC mesh for capturing accurately HF effects on some practical 3D structures such as test copper loops, power module and printed circuit layouts is used to create the pairs of rectangular volume cells characterized by a wide range of mutual distances and aspect ratios, which are in turn used to evaluate the requirements for the conditions in the new strategy. First, the new $\epsilon$-condition is set by removing the term $s_{n i}$ in the condition (5) from [14] according to the derivation given in Appendix:

$$
\text { condition }_{1}=V_{1} V_{2} \frac{d_{n 12, \max }}{R_{\min }^{3}}<\epsilon_{1} .
$$

In addition to $\epsilon_{1}$, the requirement for three more $\epsilon$-conditions is observed as explained by (7) and Fig. 5. The relative error of the improved strategy using the example from the previous section is presented in Fig. 4.
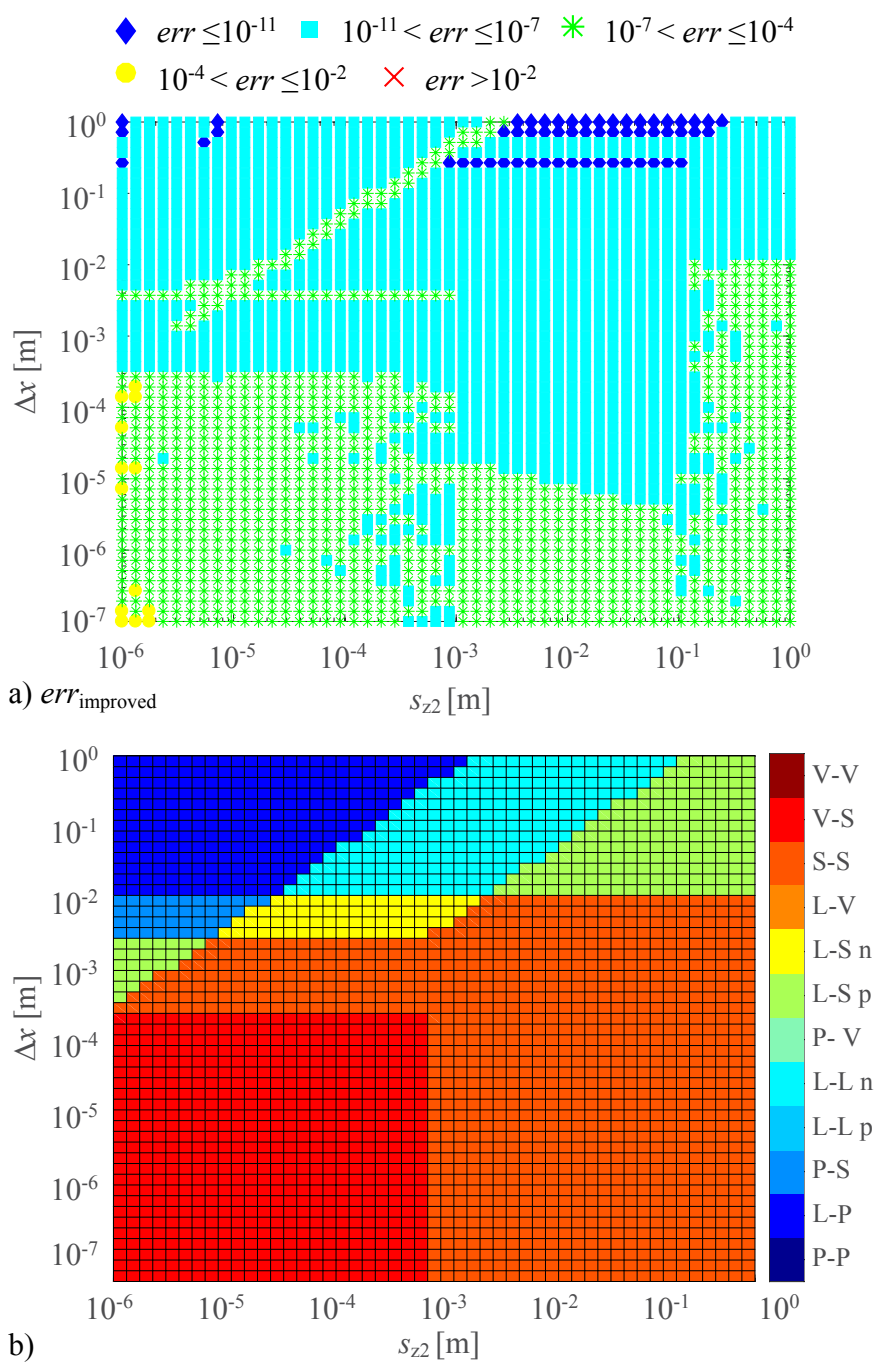

Fig. 4. Computing the mutual inductance of two specified volume cells using the proposed strategy and the derived analytical formulas for nine special cases with $\epsilon_{1}=10^{-3}, \epsilon_{2}=3 \cdot 10^{-3}, \epsilon_{3}=10^{-3}, \epsilon_{4}=3 \cdot 10^{-1}$ : a) the relative error, b) a map for the used analytical formulas, where $\mathbf{L}-\mathbf{L} \mathbf{n}$ and $\mathbf{L}-\mathbf{S} \mathbf{n}$ stands for the cases with the orthogonal, and $\mathbf{L}-\mathbf{L} \mathbf{p}$ and $\mathbf{L}-\mathbf{S} \mathbf{n}$ for the parallel orientation.

$$
\begin{aligned}
& \operatorname{condition}_{2}\left(s_{n i}\right)=s_{n i} / R_{\min }<\epsilon_{2} \\
& \operatorname{condition}_{3}\left(s_{n i}\right)=s_{n i} / \max \left(s_{x i}, s_{y i}, s_{z i}\right)<\epsilon_{3} \\
& \text { condition }_{\mathrm{A}}=\text { condition }_{1} \text { and }\left(\text { condition }_{2}\right. \text { or condition } \\
& 3
\end{aligned}
$$

As it can be seen, the relative error $\epsilon_{\text {improved }}$ is reduced below $1 \%$. The right set of $\epsilon$-conditions $\left(\epsilon_{1}=10^{-3}, \epsilon_{2}=3 \cdot 10^{-3}\right.$, 


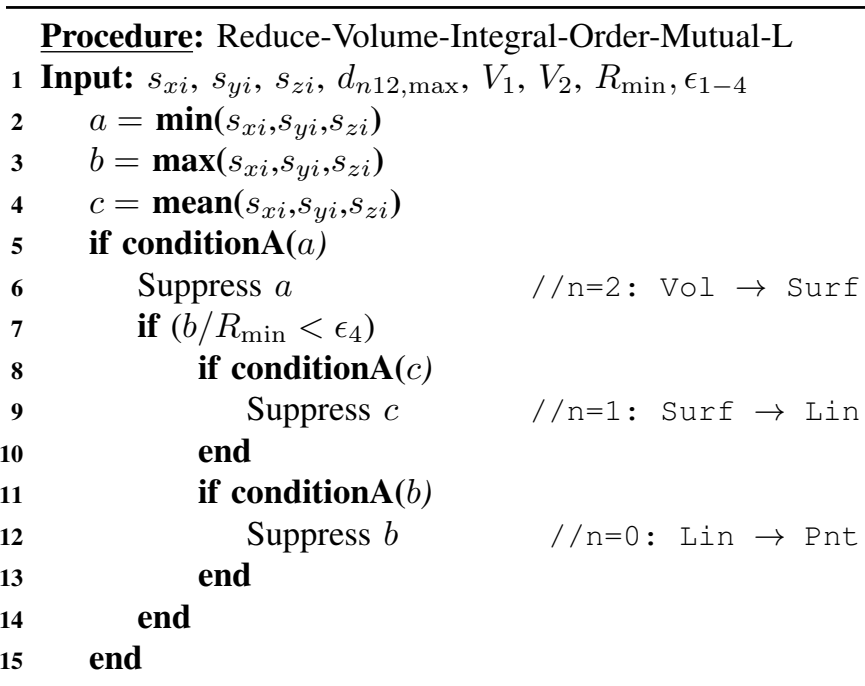

Fig. 5. Reducing the volume (three-fold) integral for the volume cell $i$ to either zero-fold, one-fold or two-fold integral depending on the cell dimensions and the distance to the other cell.

$\epsilon_{3}=10^{-3}, \epsilon_{4}=3 \cdot 10^{-1}$ ) is derived by observing a large number of volume cells with the dimensions and distances in the range of $1 \mu \mathrm{m}$ and $1 \mathrm{~m}$.

\section{Self-Inductance Computation}

In the QS-PEEC method the self-inductance is described by the diagonal elements of $\mathbf{L}_{\mathrm{p}}$ matrix, and requires the computation of the double-folded volume integral (1), where $V_{n}=V_{m}$. In [19], the analysis of four analytical formulas from [15]-[18] for the calculation of the self-inductance of a rectangular volume cell showed that the self-inductance analytical formula from [18] marked as FastHenry formula demonstrated the best behavior across a wide range of $\ell / w$, where $w$, and $\ell$ represent the width and the length of the volume cell, respectively. The relative error for the selfinductance formula from [18] is here validated for a volume cell with varying width $w$, thickness $t$, and length $\ell$ in the range between $1 \mathrm{pm}$ and $10 \mathrm{~m}$, with $n=50$ points in each direction, using the quadruple precision for calculating the reference $\mathbf{L}_{\mathrm{p}}$ values. From the results presented in Fig. 6, the analytical formula from [18] shows the relative error, err, of less than $10^{-15}$ for the all $k(w, t, \ell)$-combinations, where $k=1 . .(n \times n \times n)$.

\section{NumericAl Tests}

In this section, two numerical examples are performed to verify the accuracy and the efficiency of the proposed strategy. In the following, for the comparison, 4 different PEEC solutions are investigated:

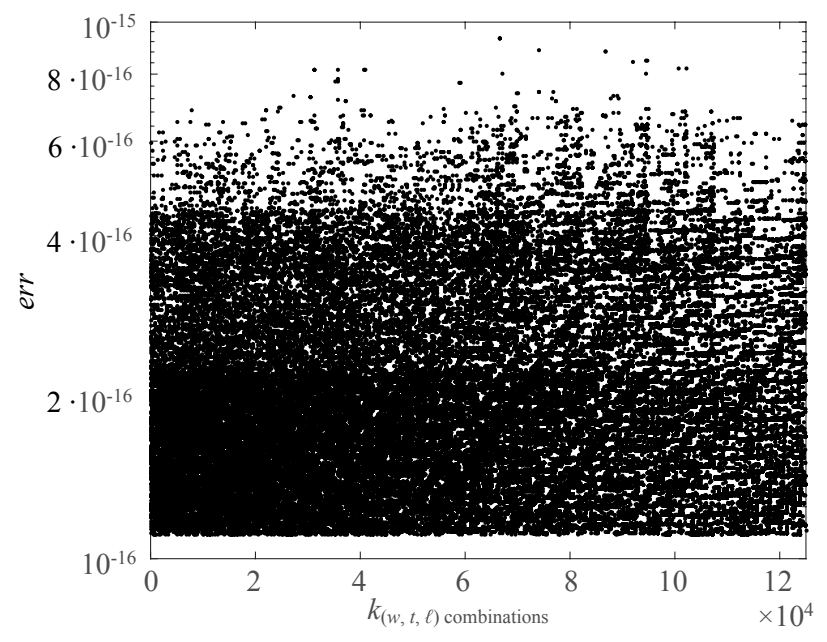

Fig. 6. Relative error for the self inductance computation of a volume cell with varying the width, $w$, the thickness, $t$, and the length, $\ell$ in the range between $1 \mathrm{pm}$ and $10 \mathrm{~m}$, with $n=50$ points in each direction, i.e. leading to $n \times n \times n$ points $k_{(w, t, \ell)}$.

- PEEC quad: the PEEC solution obtained with $\mathbf{L}_{\mathrm{p}}$ and $\mathbf{P}$ matrices calculated by using the analytical formulas from [16] in quadruple precision;

- PEEC double: the PEEC solution obtained with $\mathbf{L}_{\mathrm{p}}$ and $\mathbf{P}$ matrices calculated by using the analytical formulas from [16] in double precision;

- PEEC Song: the PEEC solution obtained with $\mathbf{L}_{\mathrm{p}}$ and $\mathbf{P}$ calculated by using the strategy described by Song et al. in [14] (in double precision);

- PEEC proposed: the PEEC solution obtained with $\mathbf{L}_{\mathrm{p}}$ and $\mathbf{P}$ matrices calculated by using the proposed strategy described in Section II-C (in double precision);

- PEEC Zhong: the PEEC solution obtained with $\mathbf{L}_{\mathrm{p}}$ calculated by using the analytical formula proposed by Zhong et al. in [13] (in double precision).

The PEEC EM solvers are developed in $\mathrm{C}++$. The quadruple precision computation is performed by using BOOST C++ libraries and the C++ Intel Math Kernel Library (Intel MKL) is used for all matrix operations. In all the simulations, the matrices coefficients are computed in parallel with 7 threads. As a measure of the accuracy, the relative error $\xi$ is defined for each solution: PEEC double $x^{\text {double }}, P E E C$ proposed $x^{\text {proposed }}$, and PEEC Zhong $x^{\text {zhong }}$, using PEEC quad solution $x^{\text {quad }}$ as the reference, where $x$ represents either a matrix or a vector of calculated results as currents and potentials:

$$
\xi=\frac{1}{N} \sum_{i}^{n} \sum_{j}^{m} \frac{\left|x_{i j}-x_{i j}^{\text {quad }}\right|}{\left|x_{i j}^{\text {quad }}\right|},
$$

where $N=n \cdot m$ is the total number of elements of $x, n=$ $m$ for the calculated matrices and $m=1$ for the vectors. The following analysis demonstrates how the calculation of $\mathbf{L}_{\mathrm{p}}$ and $\mathbf{P}$ matrices influences the calculated vectors of currents and potentials and hence, the modeled frequency-dependent $Z$, $Z(f)$. Two geometry examples with mainly inductive behavior 
are used in order to evaluate the effectiveness of the proposed approach.

\section{A. Example 1: Copper Test Loop}

The first example is a copper test loop structure described in Fig. 7. The PEEC analysis from $100 \mathrm{~Hz}$ to $100 \mathrm{MHz}$ was performed by using a non-uniform mesh (with minimum mesh size of $0.5 \mu \mathrm{m}$ in each direction) resulting in 1323 PEEC nodes and 4626 PEEC inductive branches. The minimum and maximum distances between two volume cells are $0.5 \mu \mathrm{m}$ and $6.25 \mathrm{~cm}$, respectively, while the minimum and maximum size of the volume cells are $0.5 \mu \mathrm{m}$ and $14.69 \mathrm{~mm}$. The error of the $\mathbf{L}_{\mathrm{p}}$ and $\mathbf{P}$ matrices computation for the specified PEEC solutions, PEEC double, PEEC Zhong, PEEC Song and PEEC proposed, are shown in Table I pointing out to a higher accuracy of the proposed strategy. The PEEC Song solution is calculated for different $\epsilon$ values (5) to illustrate the main challenge of the strategy proposed in [14]. Finding an $\epsilon$ value, which minimizes the error of calculating $\mathbf{L}_{\mathrm{p}}$ and $\mathbf{P}$ matrices, is not feasible without a prior knowledge of PEEC quad solution. The cpu-times spent for computing the PEEC matrices $\mathbf{L}_{\mathrm{p}}$ and $\mathbf{P}$ are summarized in Table II showing that the PEEC quad solution is highly computationally expensive. The use of the quadruple precision computation leads to a high computation time already for the case of a small problem size such as the test loop structure under analysis. In addition, the PEEC solution PEEC proposed and [14] show faster computation times as they are based on the derived analytical formulas, which are less expensive than the analytical formulas used for the volume-volume integration for $\mathbf{L}_{p}$ in [13], [16] (or surfacesurface integration for $\mathbf{P}$ ).

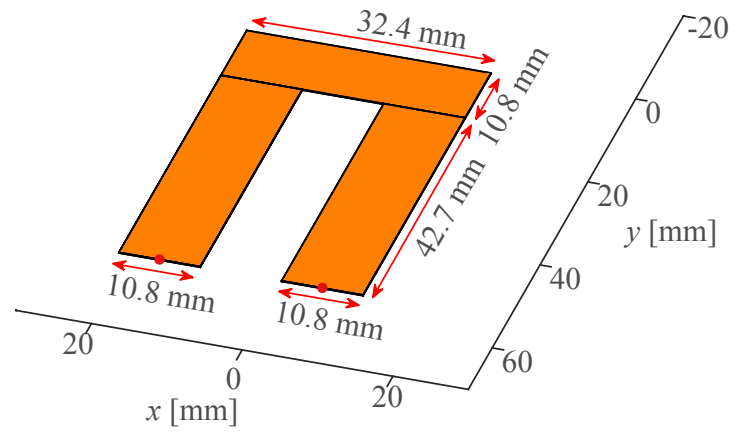

Fig. 7. First modeling example: a copper test loop structure with the thickness of $0.1 \mathrm{~mm}$ along $z$-axis.

In the next step, the accuracy of calculating two vectors containing the PEEC cell currents and node potentials, respectively, is evaluated for the PEEC solutions PEEC double, PEEC Zhong, PEEC Song and PEEC proposed. The errors are computed by using (8) in the frequency range from $100 \mathrm{~Hz}$ to $100 \mathrm{MHz}$ and presented in Fig. 8. Specifically, Fig. 8 demonstrates how the errors in $\mathbf{L}_{\mathrm{p}}$ matrix influence the calculation of the current and potential distributions. The errors in $\mathbf{L}_{\mathrm{p}}$ have a higher impact on the calculation of the current
TABLE I

RELATIVE ERROR OF $\mathbf{L}_{\mathrm{p}}$ AND $\mathbf{P}$ MATRICES COMPUTATION FOR THE TEST LOOP MODELING EXAMPLE.

\begin{tabular}{|c|c|c|}
\hline & $\mathbf{L}_{\mathrm{p}}$ & $\mathbf{P}$ \\
\hline \hline PEEC double & $1.9 \cdot 10^{7}$ & 39.2 \\
\hline PEEC Zhong & 43.06 & - \\
\hline PEEC Song $\left(\epsilon=10^{-18}\right)$ & $8.9 \cdot 10^{-3}$ & $2.0 \cdot 10^{-3}$ \\
\hline PEEC Song $\left(\epsilon=10^{-20}\right)$ & $4.9 \cdot 10^{-3}$ & $5.4 \cdot 10^{-2}$ \\
\hline PEEC Song $\left(\epsilon=10^{-21}\right)$ & $3.5 \cdot 10^{-3}$ & 0.4268 \\
\hline PEEC Song $\left(\epsilon=10^{-23}\right)$ & $2.0 \cdot 10^{-3}$ & 22.69 \\
\hline PEEC Song $\left(\epsilon=10^{-25}\right)$ & $5.75 \cdot 10^{-2}$ & 37.07 \\
\hline PEEC proposed & $1.19 \cdot 10^{-5}$ & $1.09 \cdot 10^{-6}$ \\
\hline
\end{tabular}

TABLE II

CPu-time for COMPUTing $\mathbf{L}_{\mathrm{p}}$ And $\mathbf{P}$ for the Test loop eXample.

\begin{tabular}{|c|c|c|}
\hline & $\mathbf{L}_{\mathrm{p}}$ & $\mathbf{P}$ \\
\hline \hline PEEC quad & 8 hours & 8 minutes \\
\hline PEEC double & 6 seconds & 1 second \\
\hline PEEC Zhong & 17 seconds & - \\
\hline PEEC Song & 1 second & 1 second \\
\hline PEEC proposed & 1 second & 1 second \\
\hline
\end{tabular}

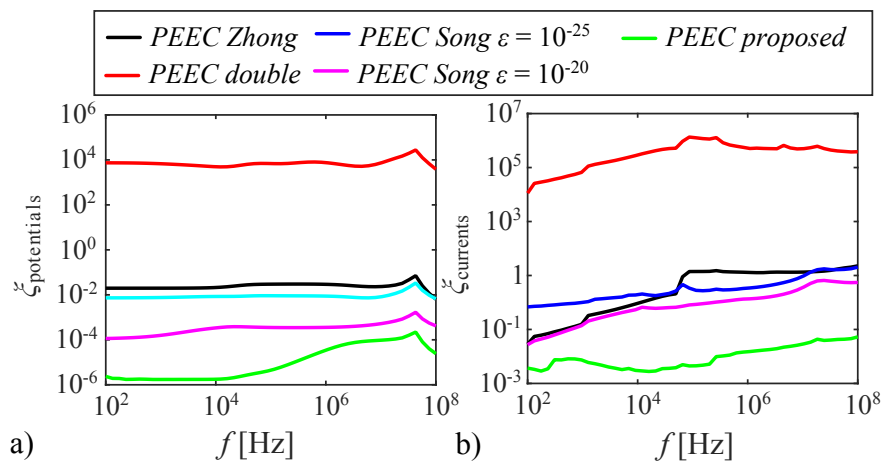

Fig. 8. $\xi$-errors of the current and potential vectors for the first modeling example computed by using (8) in the frequency range from $100 \mathrm{~Hz}$ to $100 \mathrm{MHz}$.

distribution, cf. Fig. 8b). The proposed method provides the most accurate results for the calculation of the current vector with the highest error of approx. 0.053, while the maximum errors for the PEEC Zhong and PEEC Song solutions are 22.72 and 5.52 , respectively. Current distribution is used in the post-processing step to calculate electromagnetic fields.

For the vector of potentials, the calculation error is high only for the PEEC solution, PEEC double. The potentials of the PEEC nodes are used to calculate the impedance $(Z)$ parameters by exciting the ports with current sources of $1 \mathrm{~A}$. A port for the input impedance calculation $Z_{\text {in }}(f)$ can be set between two PEEC nodes in the middle at $x z$-cross section as marked in Fig. 7. Therefore, with small differences between the vectors of potentials, it can be concluded that the errors in $\mathbf{L}_{\mathrm{p}}$ in the case of PEEC Zhong, PEEC Song and PEEC proposed do not affect significantly the calculation of $Z$ parameters in the comparison to the PEEC double solution. On the other hand, instead of the point excitations, the loop can be excited by two equipotential surfaces placed across the $x z$-cross sections. This type of excitation reduces the error of 
$Z_{\text {in }}(f)$ calculated by PEEC double, which can be explained by the dominance of the diagonal $\mathbf{L}_{\mathrm{p}}$ matrix elements for the calculation of $Z_{\text {in }}(f)$, i.e. accurately calculated self in-

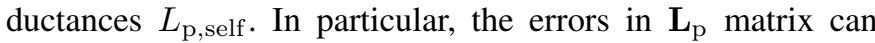
have a low impact on the calculation of $Z$ parameters due to the dominance of the other $\mathbf{L}_{\mathrm{p}}$ matrix elements accurately calculated, which depends on the modeling geometry, the mesh and the excitation ports. However, they have a higher impact on the calculation of the current distribution. In the next example, the equipotential excitation surfaces are used to show how this boundary condition affects the calculation of currents and potentials.

\section{B. Example 2: Power Busbars}

The second example of two busbars placed on a copper layer is depicted in Fig. 9. The busbars model is discretized by a non-uniform mesh with the minimum mesh size of $0.5 \mu \mathrm{m}$ in each direction, resulting in 10138 elementary surfaces (PEEC nodes) and 16628 elementary volumes (PEEC edges). The minimum and maximum distances between two volume cells are $0.354 \mu \mathrm{m}$ and $11.4 \mathrm{~cm}$, respectively, while the minimum and maximum size of the volume cells are $0.5 \mu \mathrm{m}$ and $9.15 \mathrm{~mm}$, respectively. The excitation probes for calculating the input impedance are two equipotential surfaces marked by the green areas in Fig. 9. The relative errors of all four PEEC

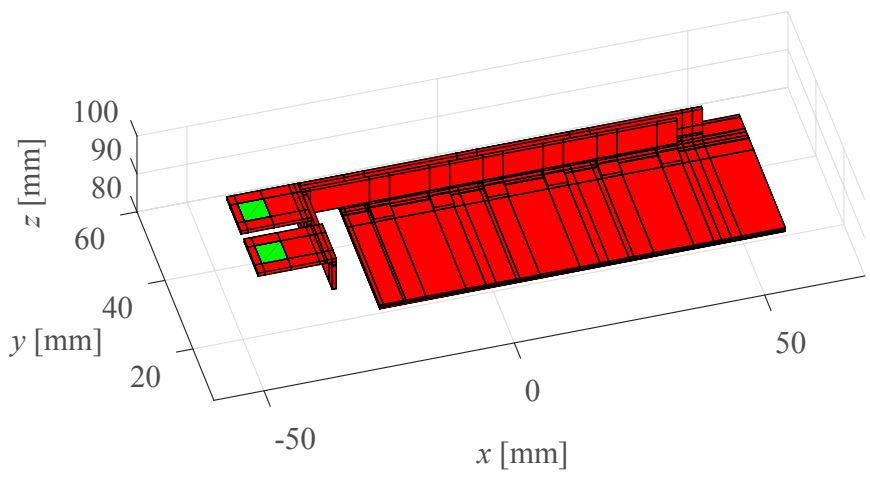

Fig. 9. Second modeling example: two busbars connected to a copper layer.

solutions, PEEC double, PEEC Zhong, PEEC Song and PEEC proposed, for the matrix $\mathbf{L}_{\mathrm{p}}$ is shown in Table III pointing out to the high accuracy of the proposed strategy. Table III contains as well as the relative error of the standard formula for calculation of off-diagonal $\mathbf{P}$ matrix elements and the relative error when extracting the $\mathbf{P}$ matrix based on the proposed strategy. For this geometry example, PEEC Song solution with $\epsilon=10^{-20}$ shows a good accuracy, however finding an optimal $\epsilon$ without having a prior knowledge of PEEC quad solution is not feasible as also demonstrated in the first example.

Three selected examples of off-diagonal $\mathbf{L}_{\mathrm{p}}$ matrix elements, i.e. the mutual inductance between two rectangular cells, for which the relative errors using the analytical formulas from [13], [14], [16] are higher than $1 \%$ are summarized in
TABLE III

RELATIVE ERROR OF $\mathbf{L}_{\mathrm{p}}$ AND $\mathbf{P}$ MATRIX CALCULATION FOR THE BUS-BARS EXAMPLE.

\begin{tabular}{|c|c|c|}
\hline & $\mathbf{L}_{\mathrm{p}}$ & $\mathbf{P}$ \\
\hline \hline PEEC double & $1.6 \cdot 10^{5}$ & 10.24 \\
\hline PEEC Zhong & $1.3 \cdot 10^{3}$ & - \\
\hline PEEC Song $\left(\epsilon=10^{-20}\right)$ & $4.2 \cdot 10^{-4}$ & $3.6 \cdot 10^{-2}$ \\
\hline PEEC proposed & $5.8 \cdot 10^{-7}$ & $1.44 \cdot 10^{-7}$ \\
\hline
\end{tabular}

Table IV, using the geometry dimensions as marked on Fig. 1. In all three selected cases from Table IV, the PEEC double solution leads to the highest error. However, the PEEC Song and PEEC Zhong do not show the same behavior. In the first and second case, the PEEC Zhong solution leads to an $\xi$ error higher than $1 \%$, while for the third case PEEC Zhong solution has a low error, which is opposite for the PEEC Song solution.

TABLE IV

THREE SELECTED EXAMPLES OF $\mathbf{L}_{\mathrm{p}}$ OFF-DIAGONAL MATRIX ELEMENTS FOR WHICH THE RELATIVE ERRORS $\xi$ USING THE ANALYTICAL FORMULAS FROM [13], [14], [16] ARE HIGHER THAN 0.01.

\begin{tabular}{|c||c|c|c|}
\hline & case 1 & case 2 & case 3 \\
\hline \hline$s x_{1}[\mathrm{~m}]$ & $10^{-6}$ & $3 \cdot 10^{-6}$ & $3 \cdot 10^{-6}$ \\
\hline$s y_{1}[\mathrm{~m}]$ & $5 \cdot 10^{-5}$ & $5 \cdot 10^{-5}$ & $5 \cdot 10^{-5}$ \\
\hline$s z_{1}[\mathrm{~m}]$ & $1.5 \cdot 10^{-4}$ & $1.5 \cdot 10^{-4}$ & $1.5 \cdot 10^{-4}$ \\
\hline$s x_{2}[\mathrm{~m}]$ & $2.59 \cdot 10^{-3}$ & $8 \cdot 10^{-4}$ & $3.38 \cdot 10^{-2}$ \\
\hline$s y_{2}[\mathrm{~m}]$ & $5 \cdot 10^{-7}$ & $2 \cdot 10^{-6}$ & $2 \cdot 10^{-6}$ \\
\hline$s z_{2}[\mathrm{~m}]$ & $4 \cdot 10^{-4}$ & $5 \cdot 10^{-7}$ & $1.5 \cdot 10^{-4}$ \\
\hline$R_{\min }[\mathrm{m}]$ & 0.092057 & 0.0801 & 0.00723 \\
\hline$L_{\mathrm{quad}}[\mathrm{H}]$ & $2.7835 \cdot 10^{-15}$ & $2.9794 \cdot 10^{-15}$ & $2.5189 \cdot 10^{-13}$ \\
\hline$\xi[13]$ & 13.76 & 11.93 & $1.02 \cdot 10^{-4}$ \\
\hline$\xi[16]$ & $6.97 \cdot 10^{3}$ & $4.15 \cdot 10^{5}$ & 0.1878 \\
\hline$\xi[14]^{*}$ & $6.21 \cdot 10^{-5}$ & $7.92 \cdot 10^{-7}$ & 0.1799 \\
\hline$\xi$ proposed & $1.15 \cdot 10^{-7}$ & $1.56 \cdot 10^{-7}$ & $4.32 \cdot 10^{-11}$ \\
\hline
\end{tabular}

$* \epsilon_{\text {condition }}=10^{-20}$

The CPU-time for computing the PEEC matrices $\mathbf{L}_{\mathrm{p}}$ and $\mathbf{P}$ is summarized in Table $\mathrm{V}$ that illustrates the importance of calculating $\mathbf{L}_{p}$ and $\mathbf{P}$ matrices in the double precision. In particular, the use of the quadruple precision computation is prohibitive also when parallel computation is exploited, while the proposed method leads to a faster computation thanks to the use of analytical formulas calculated in double precision arithmetic, c.f. Table II.

TABLE V

CPU-TIME SPENT For COMPUTING $\mathbf{L}_{\mathrm{p}}$ AND $\mathbf{P}$ FOR THE BUS-BARS EXAMPLE.

\begin{tabular}{|c|c|c|}
\hline & $\mathbf{L}_{\mathrm{p}}$ & $\mathbf{P}$ \\
\hline \hline PEEC quad & 5 days & 23 hours \\
\hline PEEC double & 75 seconds & 25 seconds \\
\hline PEEC Zhong & 231 seconds & - \\
\hline PEEC Song & 58 seconds & 23 seconds \\
\hline PEEC proposed & 34 seconds & 21 seconds \\
\hline
\end{tabular}

The average relative errors of calculating the current and potential vectors in the frequency range from $100 \mathrm{~Hz}$ to $100 \mathrm{MHz}$, based on (8), are shown in Fig. 10. The errors of the node potentials are small (less than 0.01) for all four cases 
PEEC double, PEEC Zhong, PEEC Song and PEEC proposed, and hence, the differences in the calculation of the input impedance $Z_{\text {in }}(f)$ when using different PEEC solutions are negligible. This can be explained by the effect of equipotential excitations that makes the diagonal $\mathbf{L}_{\mathrm{p}}$ elements dominant as noted in Section IV-A. On the other hand, the error of the current vector computation for the PEEC proposed solution is significantly lower than for the other three solutions. The maximum $\xi$ errors for the PEEC proposed, PEEC Song, PEEC Zhong and PEEC double solutions are 0.002, 4.02, 172.9 and 255.9 , respectively. Therefore, the proposed PEEC solution for $\mathbf{L}_{\mathrm{p}}$ and $\mathbf{P}$ matrix calculation is valuable if the current distribution has to be determined with a higher accuracy.

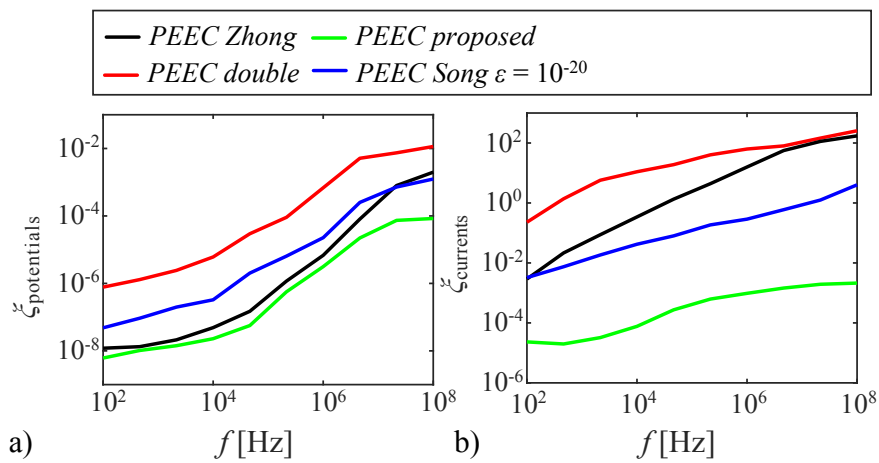

Fig. 10. $\xi$-errors of the current and potential vectors for the second modeling example computed by using (8) in the frequency range from $100 \mathrm{~Hz}$ to $100 \mathrm{MHz}$

\section{CONCLUSIONS}

The PEEC-based modeling accuracy strongly depends on the accuracy of calculated partial resistances/inductance and coefficients of potentials. The closed-form analytical formulas from literature for the calculation of partial inductances are revised systematically looking into their range of accuracy and validity for practical geometrical examples. The new developed strategy allows automatic selection of a proper analytical formula for two rectangular conductors and leads to the relative error of less than $0.1 \%$ for the extraction of parasitic inductances. Additionally, the derived analytical formulas can be useful to any other numerical method requiring double folded volume or surface integrations of the free-space Green's function over rectangular domains.

\section{APPENDIX}

\section{A. First Condition For Reducing the Order of Integrals}

A derivation of the condition for approximating the double folded volume integral (1) by a fifth order integral (4a) is explained in this section. Starting from (9), the order reduction is performed for the first cell (without loss of generality) in the dimension $\ell_{i}$, which can be one of three directions in Cartesian coordinate system $(x, y, z)$ of the $i$-th cell, where $i=1,2$.

$$
\begin{array}{r}
\int_{V_{i}} \int_{V_{j}} \frac{1}{r} d V_{i} d V_{j}=\int_{s_{n a}}^{s_{n b}} \int_{S_{i}} \int_{V_{j}} \frac{1}{r} d V_{j} d S_{i} d \ell_{i} \\
\mathcal{I}_{v-v}=\int_{s_{n a}}^{s_{n b}} \mathbf{I}_{s-v} d \ell_{i} \approx\left(s_{n b}-s_{n a}\right) \mathcal{I}_{s-v},
\end{array}
$$

where $s_{n}=s_{n b}-s_{n a}$ is the cell dimension in the suppressed direction, i.e. one of the values $s_{x i}, s_{y i}, s_{z i}$, where $i=1,2$. According to [14], this approximation is valid if $\left|\partial \mathcal{I}_{s-v} / \partial \ell_{i}\right| \rightarrow 0$ or $s_{n} \rightarrow 0$, which is combined in the condition $\left|s_{n} \cdot \partial \mathcal{I}_{s-v} / \partial \ell_{i}\right|<\epsilon$, where $\epsilon$ is an extremely small positive value. A condition to approximate six-fold integral by means of five-fold integral (i.e. suppressing the dimension $\ell_{i}$ of the $i$-th cell) can be derived from (10)-(11) assuming $s_{n}=$ $s_{x 1}$ (without loss of generality).

$$
\begin{aligned}
\left|\frac{\partial \mathcal{I}_{s-v}}{\partial \ell_{i}}\right| & =\int_{y_{1}} \int_{z_{1}} \int_{x_{2}} \int_{y_{2}} \int_{z_{2}}\left|\frac{\partial F\left(x_{1}\right)}{\partial x_{1}}\right| d y_{1} d z_{1} d x_{2} d y_{2} d z_{2} \\
& =\int_{y_{1}} \int_{z_{1}} \int_{x_{2}} \int_{y_{2}} \int_{z_{2}} \frac{\left|x_{1}-x_{2}\right|}{R^{3}} d y_{1} d z_{1} d x_{2} d y_{2} d z_{2}
\end{aligned}
$$

$$
\left|s_{n} \frac{\partial \mathcal{I}_{s-v}}{\partial \ell_{i}}\right| \leq \underbrace{s_{x 1} \frac{\max \left|x_{1}-x_{2}\right|}{R_{\min }^{3}} s_{y 1} s_{z 1} s_{x 2} s_{y 2} s_{z 2}<\epsilon}_{\text {condition }_{1}: V_{1} V_{2} \frac{d_{x, \max }}{R_{\min }^{3}}<\epsilon}
$$

using (12),

$$
\int_{a}^{b} f(x) d x \leq(b-a) \cdot \max (f(x))
$$

where $F\left(x_{1}\right)=1 / \sqrt{\left(x_{1}-x_{2}\right)^{2}+\left(y_{1}-y_{2}\right)^{2}+\left(z_{1}-z_{2}\right)^{2}}$ and $R=\sqrt{\left(x_{1}-x_{2}\right)^{2}+\left(y_{1}-y_{2}\right)^{2}+\left(z_{1}-z_{2}\right)^{2}}, V_{1}$ and $V_{2}$ are the volumes of two cells, $d_{x, \max }$ is the maximum distance between two cells in the $x$-direction, and $R_{\min }$ is the minimum distance between two cells.

\section{B. Surface-Line}

Interaction between surfaces and lines can be divided in two sub-cases: parallel and orthogonal. In the first case a line is orthogonal to the normal of a surface, while for the second case it is parallel. The integral to be computed is both cases is

$$
\mathcal{I}_{s-\ell}=\int_{S_{j}} \int_{\ell_{i}} \frac{1}{r} d \ell_{i} d S_{j}
$$

1) First Case: The solution of (13) for the case the line is orthogonal to the normal of the surface is derived in the following by observing a rectangular surface defined by two nodes with the coordinates $\left(x_{j 1}, y_{j 1}, z_{j}\right)$ and $\left(x_{j 2}, y_{j 2}, z_{j}\right)$, the length $\left(x_{j 2}-x_{j 1}\right)$ and width $\left(y_{j 2}-y_{j 1}\right)$, while $\ell_{i}$ is a line defined between the nodes $\left(x_{i 1}, y_{i}, z_{i}\right)$ and $\left(x_{i 2}, y_{i}, z_{i}\right)$. 


$$
\mathcal{I}_{s-\ell}=\sum_{c_{1}=1}^{4} \sum_{c_{2}=1}^{2} \sum_{h=1}^{4}(-1)^{c_{1}+c_{2}} \gamma_{h}\left(c_{1}, c_{2}\right)
$$

with

$$
\begin{aligned}
& \gamma_{1}=x_{c_{1}} y_{c_{2}} \log \left(x_{c_{1}}+R\left(c_{1}, c_{2}\right)\right) \\
& \gamma_{2}=\frac{1}{2}\left(x_{c_{1}}^{2}-z^{2}\right) \log \left(y_{c_{2}}+R\left(c_{1}, c_{2}\right)\right) \\
& \gamma_{3}=-x_{c_{1}} z \tan ^{-1}\left(\frac{x_{c_{1}} y_{c_{2}}}{z R\left(c_{1}, c_{2}, y_{1}, c_{3}, z_{1}, z_{2}\right)}\right) \\
& \gamma_{4}=-\frac{1}{2} y_{c_{2}} R\left(c_{1}, c_{2}\right) \\
& R\left(c_{1}, c_{2}\right)=\sqrt{x_{c_{1}}^{2}+y_{c_{2}}^{2}+z^{2}}
\end{aligned}
$$

and

$$
\begin{array}{ll}
x_{1}=x_{j 2}-x_{i 2} & x_{2}=x_{j 2}-x_{i 1} \\
x_{2}=x_{j 1}-x_{i 1} & x_{2}=x_{j 1}-x_{i 2} \\
y_{1}=y_{j 1}-y_{i} & y_{2}=y_{j 2}-y_{i} \\
z=z_{j}-z_{i} . &
\end{array}
$$

2) Second Case: The solution of (13) for the case the line is parallel to the normal of the surface is derived in the following by observing a rectangular surface $S_{j}$ defined by two nodes with the coordinates $\left(x_{j}, y_{j 1}, z_{j 1}\right)$ and $\left(x_{j}, y_{j 2}, z_{j 2}\right)$, the length $\left(y_{j 2}-y_{j 1}\right)$ and width $\left(z_{j 2}-z_{j 1}\right)$, while $\ell_{i}$ is a line defined between the nodes $\left(x_{i 1}, y_{i}, z_{i}\right)$ and $\left(x_{i 2}, y_{i}, z_{i}\right)$.

$$
\mathcal{I}_{s-\ell}=\sum_{c_{1}=1}^{2} \sum_{c_{2}=1}^{2} \sum_{c_{3}=1}^{2} \sum_{h=1}^{6}(-1)^{c_{1}+c_{2}+c_{3}} \gamma_{h}\left(c_{1}, c_{2}, c_{3}\right)
$$

with

$$
\begin{aligned}
& \gamma_{1}=y_{c_{2}} z_{c_{3}} \log \left(x_{c_{1}}+R\left(c_{1}, c_{2}, c_{3}\right)\right) \\
& \gamma_{2}=x_{c_{1}} z_{c_{3}} \log \left(y_{c_{2}}+R\left(c_{1}, c_{2}, c_{3}\right)\right) \\
& \gamma_{3}=y_{c_{2}} x_{c_{1}} \log \left(z_{c_{3}}+R\left(c_{1}, c_{2}, c_{3}\right)\right) \\
& \gamma_{4}=-\frac{1}{2} z_{c_{3}}^{2} \tan ^{-1}\left(\frac{x_{c_{1}} y_{c_{2}}}{z_{c_{3}} R\left(c_{1}, c_{2}, c_{3}\right)}\right) \\
& \gamma_{5}=-\frac{1}{2} y_{c_{2}}^{2} \tan ^{-1}\left(\frac{x_{c_{1}} z_{c_{3}}}{y_{c_{2}} R\left(c_{1}, c_{2}, c_{3}\right)}\right) \\
& \gamma_{6}=-\frac{1}{2} x_{c_{1}}^{2} \tan ^{-1}\left(\frac{y_{c_{2}} z_{c_{3}}}{x_{c_{1}} R\left(c_{1}, c_{2}, c_{3}\right)}\right) \\
& R\left(c_{1}, c_{2}\right)=\sqrt{x_{c_{1}}^{2}+y_{c_{2}}^{2}+z_{c_{3}}^{2}}
\end{aligned}
$$

and

$$
\begin{array}{ll}
x_{1}=x_{j}-x_{i 2} & x_{2}=x_{j}-x_{i 1} \\
y_{1}=y_{j 2}-y_{i} & y_{2}=y_{j 1}-y_{i} \\
z=z_{j 2}-z_{i} & z_{2}=z_{j 1}-z_{i} .
\end{array}
$$

\section{Line-Line}

When each cell can be approximated by a line, there are two different cases which may occur: parallel and orthogonal lines. The integral to be solved in both cases is given by

$$
\mathcal{I}_{\ell-\ell}=\int_{\ell_{j}} \int_{\ell_{i}} \frac{1}{r} d \ell_{i} d \ell_{j}
$$

1) Parallel Lines: The solution of (20) for the case of parallel lines is given in the following by observing a line $\ell_{i}$ defined by the nodes $\left(x_{i 1}, y_{i}, z_{i}\right)$ and $\left(x_{i 2}, y_{i}, z_{i}\right)$ and a line $\ell_{j}$ defined by the nodes $\left(x_{j 1}, y_{j}, z_{j}\right)$ and $\left(x_{j 2}, y_{j}, z_{j}\right)$.

$\mathcal{I}_{\ell-\ell}=\sum_{c_{1}=1}^{4}(-1)^{c_{1}}\left[x_{c_{1}} \log \left(x_{c_{1}}+\sqrt{\rho+x_{c_{1}}}\right)-\sqrt{\rho+x_{c_{1}}}\right]$

where

$$
\rho=\sqrt{\left(y_{i}-y_{j}\right)^{2}+\left(z_{i}-z_{j}\right)^{2}}
$$

and

$$
\begin{array}{r}
x_{1}=x_{i 2}-x_{j 2}, \quad x_{2}=x_{i 2}-x_{j 1}, \\
x_{3}=x_{i 1}-x_{j 1}, \quad x_{4}=x_{i 1}-x_{j 2}
\end{array}
$$

if $\rho \neq 0$, or

$$
\begin{gathered}
x_{1}=\left|x_{i 2}-x_{j 2}\right|, x_{2}=\left|x_{i 2}-x_{j 1}\right|, \\
x_{3}=\left|x_{i 1}-x_{j 1}\right|, x_{4}=\left|x_{i 1}-x_{j 2}\right|
\end{gathered}
$$

if $\rho=0$. It is worth to notice that (21) becomes singular when the lines are overlapped for more than one point.

2) Orthogonal lines: In the orthogonal case the solution of (20) two lines are observed: $\ell_{i}$ defined by the nodes $\left(x_{i 1}, y_{i}, z_{i}\right)$ and $\left(x_{i 2}, y_{i}, z_{i}\right)$ and a line $\ell_{j}$ defined by the nodes $\left(x_{j}, y_{j 1}, z_{j}\right)$ and $\left(x_{j}, y_{j 2}, z_{j}\right)$.

$$
\mathcal{I}_{\ell-\ell}=\sum_{c_{1}=1}^{2} \sum_{c_{2}=1}^{2} \sum_{h=1}^{3}(-1)^{c_{1}+c_{2}+1} \gamma_{h}\left(c_{1}, c_{2}\right)
$$

with

$$
\begin{aligned}
& \gamma_{1}=x_{c_{1}} \log \left(y_{c_{2}}+R\left(c_{1}, c_{2}\right)\right) \\
& \gamma_{2}=y_{c_{2}} \log \left(x_{c_{1}}+R\left(c_{1}, c_{2}\right)\right) \\
& \gamma_{3}=-z \tan ^{-1}\left(\frac{x_{c_{1}} y_{c_{2}}}{z R\left(c_{1}, c_{2}\right)}\right) \\
& R\left(c_{1}, c_{2}\right)=\sqrt{x_{c_{1}}^{2}+y_{c_{2}}^{2}+z^{2}}
\end{aligned}
$$

and

$$
\begin{array}{ll}
x_{1}=x_{i 2}-x_{j} & x_{2}=x_{i 1}-x_{j} \\
y_{1}=y_{i}-y_{j 1} & y_{2}=y_{i}-y_{j 2} \\
z=z_{i}-z_{j} . &
\end{array}
$$




\section{Tips for integrals evaluation}

The solution for the singularities of integrals in the form of (4) can be found in [22]. In this section we summarize the formulas helpful for solving the singularities of the integrals given by (14)-(25). The singularity of type $x \log \left(\sqrt{x^{2}+y^{2}+z^{2}}\right)$ is solved using (28),

$$
\lim _{x \rightarrow 0}\left[x \log \left(\sqrt{x^{2}+y^{2}+z^{2}}\right)\right]=0 .
$$

The function $x^{2} \tan ^{-1}\left(\frac{y z}{x \sqrt{x^{2}+y^{2}+z^{2}}}\right)$ is evaluated as $x|x| \arctan 2\left(\frac{y z}{|x| \sqrt{x^{2}+y^{2}+z^{2}}}\right)$, where the function $\arctan 2$ is defined by (29), with $\tan ^{-1}(q)$ representing the inverse tangent of $q$ computed in the range $[-\pi / 2, \pi / 2]$.

$\arctan 2\left(\frac{k}{m}\right)= \begin{cases}\tan ^{-1}\left(\frac{k}{m}\right) & \text { if } m>0 \\ \tan ^{-1}\left(\frac{k}{m}\right)+\pi & \text { if } k \geq 0 \& m<0 \\ \tan ^{-1}\left(\frac{k}{m}\right)-\pi & \text { if } k<0 \& m<0 \\ \frac{\pi}{2} & \text { if } k>0 \& m=0 \\ -\frac{\pi}{2} & \text { if } k<0 \& m=0\end{cases}$

\section{REFERENCES}

[1] T. Reimann and M. Scherf, "Frequency optimum of semiconductor technologies and state-of-the-art magnetic components on smps," in Proc. of 10th International Conference on Integrated Power Electronics Systems (CIPS), March 2018, pp. 1-8.

[2] ANSYS, "Ansys electromagnetics," Jul. 2019. [Online]. Available: https://www.ansys.com/products/electronics

[3] CST, "Cst studio suite," Jul. 2019. [Online]. Available: https: //www.3ds.com/products-services/simulia/products/cst-studio-suite/

[4] COMSOL, "Comsol electromagnetics modules," Jul. 2019. [Online]. Available: https://www.comsol.com/products

[5] D. Popescu and M. Treiber, "Broadband TCAD mixed-mode simulation framework for predictive modeling of fast dynamic switching events," in Proc. of 31st Int. Symp. on Power Semiconductor Devices and ICs ISPSD, 2019, pp. 327-330.

[6] A. E. Ruehli, "Equivalent circuit models for three dimensional multiconductor systems," IEEE Trans. on Microwave Theory and Techniques, vol. MTT-22, no. 3, pp. 216-221, Mar. 1974.

[7] A. E. Ruehli, G. Antonini, and L. Jiang, Circuit Oriented Electromagnetic Modeling Using the PEEC Techniques. Wiley-IEEE Press, 2017.

[8] A. E. Ruehli, G. Antonini, J. Esch, J. Ekmana, A. Mayo, and A. Orlandi, "Non-orthogonal PEEC formulation for time and frequency domain EM and circuit modeling," IEEE Trans. on Electromagnetic Compatability, vol. 45, no. 2, pp. 167-176, May 2003.

[9] J. Ekman, G. Antonini, A. Orlandi, and A. E. Ruehli, "Impact of partial element accuracy on peec model stability," IEEE Trans. Electromagn. Compat., vol. 48, no. 1, pp. 19-32, Feb 2006.

[10] Y. Hackl, P. Scholz, W. Ackermann, and T. Weiland, "Multifunction approach and specialized numerical integration algorithms for fast inductance evaluations in nonorthogonal peec systems," IEEE Transactions on Electromagnetic Compatibility, vol. 57, no. 5, pp. 1155-1163, 2015.

[11] A. Musing, J. Ekman, and J. W. Kolar, "Efficient calculation of nonorthogonal partial elements for the peec method," IEEE Transactions on Magnetics, vol. 45, no. 3, pp. 1140-1143, 2009.

[12] R. Y. Zhang, J. K. White, and J. G. Kassakian, "Fast simulation of complicated 3-d structures above lossy magnetic media," IEEE Transactions on Magnetics, vol. 50, no. 10, pp. 1-16, Oct 2014.
[13] Guoan Zhong and Cheng-Kok Koh, "Exact closed-form formula for partial mutual inductances of rectangular conductors," IEEE Transactions on Circuits and Systems I: Fundamental Theory and Applications, vol. 50, no. 10, pp. 1349-1352, Oct 2003.

[14] Z. Song, F. Duval, D. Su, and A. Louis, "Stable partial inductance calculation for partial element equivalent circuit modeling," Applied Computational Electromagnetics Society (ACES) Journal, vol. 25, no. 9, 2010., vol. 25, pp. 738-749, 2010.

[15] F. W. Grover, Inductance calculations: Working formulas and tables. Dover, 1962.

[16] C. Hoer, C. Love, "Exact Inductance Equations for Rectangular Conductors With Applications to More Complicated Geometries," J. Research National Bureau of Standards-C. Engineering Instrumentation, vol. 69, no. C, pp. 127-137, 1965.

[17] A. E. Ruehli, "Inductance calculations in a complex integrated circuit environment," IBM Journal of Research and Development, vol. 16, no. 5, pp. 470-481, Sep. 1972.

[18] Ruey-Beei Wu, Chien-Nan Kuo, and K. K. Chang, "Inductance and resistance computations for three-dimensional multiconductor interconnection structures," IEEE Transactions on Microwave Theory and Techniques, vol. 40, no. 2, pp. 263-271, Feb 1992.

[19] H. Kim and C. C.-P. Chen, "Be careful of self and mutual inductance formulae," Technical Report University of Wisconsin-Madison, Tech. Rep., 2001.

[20] Z. Piatek, B. Baron, T. Szczegielniak, D. Kusiak, and A. Pasierbek, "Exact closed form formula for mutual inductance of conductors of rectangular cross section," Przeglad Elektrotechniczny, vol. 89, no. 3a, pp. 61-64, 2013.

[21] Z. Piatek and B. Baron, "Exact closed form formula for self inductance of conductor of rectangular cross section," Progress In Electromagnetics Research, vol. 26, pp. 225-236, 2012.

[22] D. Romano and G. Antonini, "Quasi-static partial element equivalent circuit models of linear magnetic materials," IEEE Transactions on Magnetics, vol. 51, no. 7, pp. 1-15, 2014.

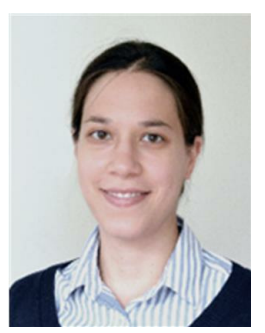

Ivana Kovăcević-Badstübner received the $\mathrm{Ph} . \mathrm{D}$. degree from the ETH Zurich, Switzerland, in 2012. From 2008 to 2015, she worked in the Power Electronics Systems (PES) Laboratory at ETH Zurich focusing on the prediction of electromagnetic behavior of power electronics systems based on the developed numerical techniques and the lifetime modeling of power semiconductor modules. In March 2016, she joined the Advanced Power Semiconductor (APS) Laboratory at ETH Zurich. Her research interests include novel packaging technologies for $\mathrm{SiC}$ power devices, the optimization of power module layout with respect to electromagnetic interference, and multi-domain modeling of power semiconductor devices and their modules.

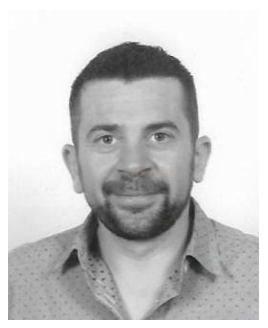

Daniele Romano was born in Campobasso, Italy, in 1984. He received the Laurea degree in computer science and automation engineering in 2012 from University of L'Aquila, L'Aquila, Italy. $\mathrm{He}$ received the Ph.D. degree in 2018. Since 2012 he has been with the UAq EMC Laboratory, University of L'Aquila, focusing on EMC modeling and analysis, algorithm engineering and speed-up techniques applied to EMC problems. 


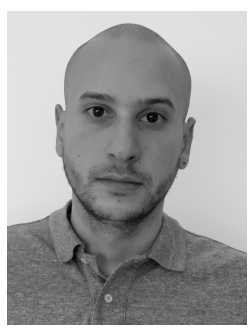

Luigi Lombardi received the Laurea (MD) in electronic engineering and the $\mathrm{PhD}$, both cum laude, from University of L'Aquila, L'Aquila, Italy. In early 2018 he was a visiting researcher with the department of electronics in Carleton University, Ottawa, Ontario, Canada. Since November 2018 he works as a design engineer with the Non Volatile Memory (NVM) design team at Micron Semiconductor, Avezzano, AQ, Italy.

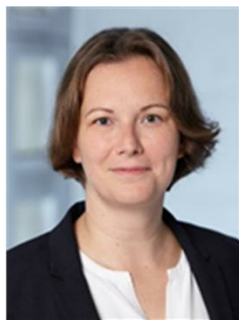

Ulrike Grossner earned the Dipl.-Phys. and the Dr. rer. nat. degree from the Friedrich-SchillerUniversity Jena, Germany in 1997 and 2000, respectively. In 2014, she was appointed Full Professor at ETH Zurich, Switzerland, where she established the Advanced Power Semiconductor Laboratory (APS), working on devices and packaging for advanced power semiconductors.

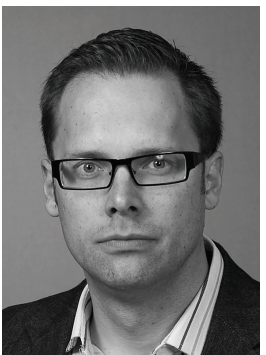

Jonas Ekman was born in Boden, Sweden, in 1972. $\mathrm{He}$ received the Ph.D. degree in electrical engineering from Luleå University of Technology, Sweden, in 2003. From 2003 to 2007, he was working as a Researcher at Luleå University of Technology. During 2005 and 2006, he did his postdoctoral research in full-wave, time domain, PEEC modelling at the EMC Laboratory, University of L'Aquila, Italy. In 2014 he was appointed Professor at Luleå University of Technology. His research interests are in computational electromagnetics, in particular, the use of the PEEC method for realistic electromagnetic modelling.

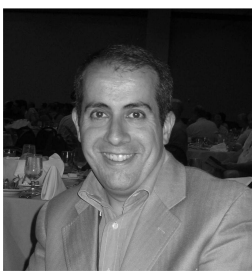

Giulio Antonini (M94 - SM05) received the Laurea degree (cum laude) in electrical engineering from the University of L'Aquila, L'Aquila, Italy, in 1994 and the Ph.D. degree in electrical engineering from University of Rome "La Sapienza" in 1998. Since 1998, he has been with the UAq EMC Laboratory, University of L'Aquila, where he is currently a Professor. He has coauthored the book "Circuit Oriented Electromagnetic Modeling Using the PEEC Techniques", (Wiley-IEEE Press, 2017). His scientific interests are in the field of computational electromagnetics. 